\title{
Adult T-cell Leukemia/Lymphoma in which the Pathohistological Diagnosis was Identical to That of Ki-1 Positive Anaplastic Large Cell Lymphoma
}

\author{
Tsutomu Takahara, Kohsuke Masutani, Eiji Kajiwara, Seizo Sadoshima, Masahiro Misago*, \\ Yasuyuki SASAGURI** and Kaoru ONOYAMA
}

\begin{abstract}
A 65-year-old man developed severe lumbago and a loss of appetite two months before presentation. A computerized tomograph at admission revealed soft tissue masses destroying the Th12, L4 and L5 vertebral bones. We diagnosed the lesions to be metastatic bone tumors, but the primary focus could not be determined. Just after the irradiation treatment, abnormal lymphocytes were detected in the peripheral blood cells. Under the suspicion of adult T-cell leukemia/ lymphoma (ATL), we thus performed a lymph node biopsy. The specimens were histologically composed of Ki-1 positive anaplastic large cell lymphoma (ALCL). The lymphoma cells demonstrated a biclonal integration of HTLV-1 proviral DNA. After 6 cycles of chemotherapy, the patient has demonstrated a partial and favorable remission from ATL.
\end{abstract}

(Internal Medicine 38: 824-828, 1999)

Key words: adult T-cell leukemia/lymphoma (ATL), anaplastic large cell lymphoma (ALCL), bone destruction, extranodal tumor, combination chemotherapy

\section{Introduction}

Adult T-cell leukemia/lymphoma (ATL) was first reported by Takatsuki et al in 1977 (1). Stein et al identified anaplastic large cell lymphoma (ALCL) based on the expression of CD30 (Ki-1) and intrasinusoidal growth patterns (2). ALCL is now regarded as a distinct clinicopathologic entity and has been included in the Revised European-American lymphoma (REAL) classification (3). CD30 ligand (CD30L) is a transmembrane protein that belongs to the tumor necrosis factor (TNF) family (4), which is rarely observed in ATL cases with HTLV-1 proviral DNA. Here, we report a case of ATL in which the pathohistological diagnosis was identical to that of Ki-1 positive ALCL, and who also demonstrated a partial remission after repeated chemotherapy.

For editorial comment, see also p 757.

\section{Case Report}

A 65-year-old man suffered from severe left back pain and a loss of appetite of two months duration. In the month before admission, he had lost $10 \mathrm{~kg}$. On June 2 1997, he was examined by an orthopedist, and L5 lytic bone destruction was observed on an X-ray examination. The patient's life history and family history were unremarkable. At admission on June 10, 1997 , his blood pressure was $122 / 76 \mathrm{mmHg}$, and his body temperature was $37.5^{\circ} \mathrm{C}$. A physical examination revealed slightly enlarged lymph nodes on his bilateral neck and inguinal regions. No heart murmur was audible and his breath sounds were vesicular. No hepatosplenomegaly nor mass lesions were evident in the abdomen. No cutaneous lesions such as cutaneous tumors or hemangioma were found. The findings of a neurological examination were all normal. Table 1 illustrates the laboratory data at admission. The white blood cell (WBC) count was $9,900 / \mathrm{mm}^{3}$ with a normal hemogram and the fraction of WBC was also normal. The platelet count was elevated. The C-reactive protein (CRP) level was also high. A biochemical examination showed the alkaline phosphatase (ALP) and lactate dehydrogenase (LDH) levels to be slightly increased, but the concentration of serum calcium was normal. Figure 1 shows the L5 bone on a computed tomograph (CT) at admission. A large tumor with $\mathrm{L} 5$ bone destruction was seen to compress his

From the Division of Internal Medicine, Nippon Steel Yawata Memorial Hospital, Kitakyushu, *Clinical Hematology and **the Second Department of Pathology, School of Health Sciences, University of Occupational and Environmental Health, Kitakyushu

Received for publication June 1, 1998; Accepted for publication June 5, 1999

Reprint requests should be adressed to Dr. Tsutomu Takahara, the Division of Internal Medicine, Nippon Steel Yawata Memorial Hospital, 1-1-1, Harunomachi, Yawatahigashiku, Kitakyushu 805-0050 
ATL and Ki-1 Positive ALCL

Table 1. Laboratory Data on Admission

\begin{tabular}{lrlr}
\hline Peripheral blood & \multicolumn{3}{c}{ Blood Chemistry } \\
White blood cell count & $9,900 / \mathrm{mm}^{3}$ & Total protein & $7.1 \mathrm{~g} / \mathrm{dl}$ \\
Neutrophil & $59.6 \%$ & Albumin & $3.8 \mathrm{~g} / \mathrm{dl}$ \\
Eosinophil & $0.3 \%$ & Total bilirubin & $0.3 \mathrm{mg} / \mathrm{dl}$ \\
Basophil & $0.3 \%$ & Asparate aminotransferase & $17 \mathrm{IU} / l$ \\
Monocyte & $7.7 \%$ & Alanine aminotransferase & $16 \mathrm{IU} / l$ \\
Lymphocyte & $32.1 \%$ & Alkaline phosphatase & $397 \mathrm{IU} / l$ \\
Red blood cell count & $403 \times 10^{4} / \mathrm{mm}^{3}$ & Lactate dehydrogenase & $563 \mathrm{IU} / l$ \\
Hemoglobin & $12.8 \mathrm{~g}^{3} / \mathrm{dl}$ & Total cholesterol & $119 \mathrm{mg} / \mathrm{dl}$ \\
Platelet & $49.0 \times 10^{4} / \mathrm{mm}^{3}$ & Glucose & $90 \mathrm{mg} / \mathrm{dl}$ \\
Serological test & & $\mathrm{Na}$ & $143 \mathrm{mEq} / l$ \\
C-reactive protein & $4.5 \mathrm{mg} / \mathrm{dl}$ & $\mathrm{K}$ & $4.7 \mathrm{mEq} / l$ \\
Hepatitis B surface antigen & $(-)$ & $\mathrm{Cl}$ & $106 \mathrm{mEq} / l$ \\
Hepatitis C antibody & $(+)$ & $\mathrm{Ca}$ & $8.7 \mathrm{mg} / \mathrm{dl}$ \\
\hline
\end{tabular}

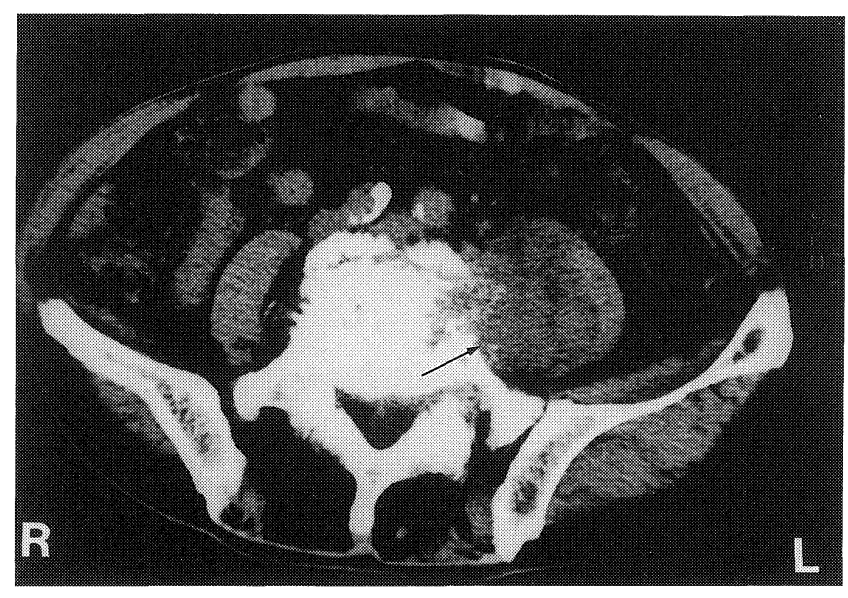

Figure 1. An abdominal CT scan on admission. A large tumor with L5 bone destruction compresses the left psoas to the left (arrow).

left psoas to the left. T2-weighted magnetic resonance imaging (MRI) showed high signal areas in the Th12, L4 and L5 regions, suggestive of tumor invasion. The spinal canal in such areas was seen to be partly compressed. He was thus diagnosed to have metastatic vertebral bone tumors, but we could not confirm the primary focus inspite of intensive examinations. From June 17 to July 7, irradiation for Th12, L4 and L5 was carried out, and his body temperature transiently decreased to a normal range, and the LDH level also decreased from 736 IU $/ l$ to $445 \mathrm{IU} / l$. Just after the irradiation treatment, we detected abnormal lymphocytes resembling flower cells in the peripheral blood. Further examinations which led us to suspect ATL indicated the anti HTLV-1 antibody to be positive (7.9 Index) while the soluble IL-2 receptor (CD25) was also abnormally high $(7,670 \mathrm{U} / \mathrm{ml})$. Bone marrow aspiration failed to show any involvement by these cells. At this time, his superficial lymph nodes were found to be enlarged. As a result, we performed a biopsy on his right neck lymph node. A histologi- cal examination showed the lymph node to have expanded to the paracortical areas and also atrophy of lymph follicles. Large abnormal cells diffusely proliferated and they had convoluted round nuclei, large nucleoli and basophilic cytoplasms (Fig. 2). On immunohistochemical staining, these abnormal cells were positive for $\mathrm{Ki}-1$ (Fig. 3) and Ber-H2. Other surface markers were CD3+, CD15-, CD20-, CD45+, CD45RO+, HLADR-, and EMR-. The materials obtained by biopsy showed the biclonal integration of HTLV-1 proviral DNA by the Southern blot hybridization method (Fig. 4). We therefore, diagnosed the primary disease to be ATL lymphoma type (stage IV B) in which the pathohistological diagnosis was identical to that of Ki-1 positive ALCL. After irradiation treatment, he again had a high temperature and the LDH rapidly began to increase. He also felt a loss of sensitivity in his lower extremities (Fig. 5). Finally, he could not stand up. This was thought to be due to bilateral iliac nerve compression by the tumors on the vertebrae. On July 28, we started combination chemotherapy. The patient received a regimen of repeated chemotherapy termed THP-CEP ie, pirarubicin (40 mg/day $\times 1$ day), cyclophosphamide (500 mg/day $\times 1$ day), etoposide (100 mg/day $\times 1$ day), and prednisolone $(40 \mathrm{mg} /$ day $\times 4$ days). The chemotherapy proved to be quite successful. After receiving 6 cycles of the therapeutic regimen over 5 months, the swelling of his superficial lymph nodes disappeared, the Th12, L4 and L5 masses remarkably decreased in size, and the dysfunction in the lower extremities almost completely improved. He was discharged on December 29 . He still receives chemotherapy treatment once a month as an outpatient and his condition remains good.

\section{Discussion}

We herein reported a case of adult T-cell leukemia/lymphoma in which the pathohistological diagnosis was identical to that of $\mathrm{Ki}-1$ positive anaplastic large cell lymphoma, who showed a favorable response to chemotherapy. Takatsuki et al first described adult T-cell leukemia/lymphoma (ATL) by HTLV infection in Japan (1). Stein et al identified anaplastic large cell 

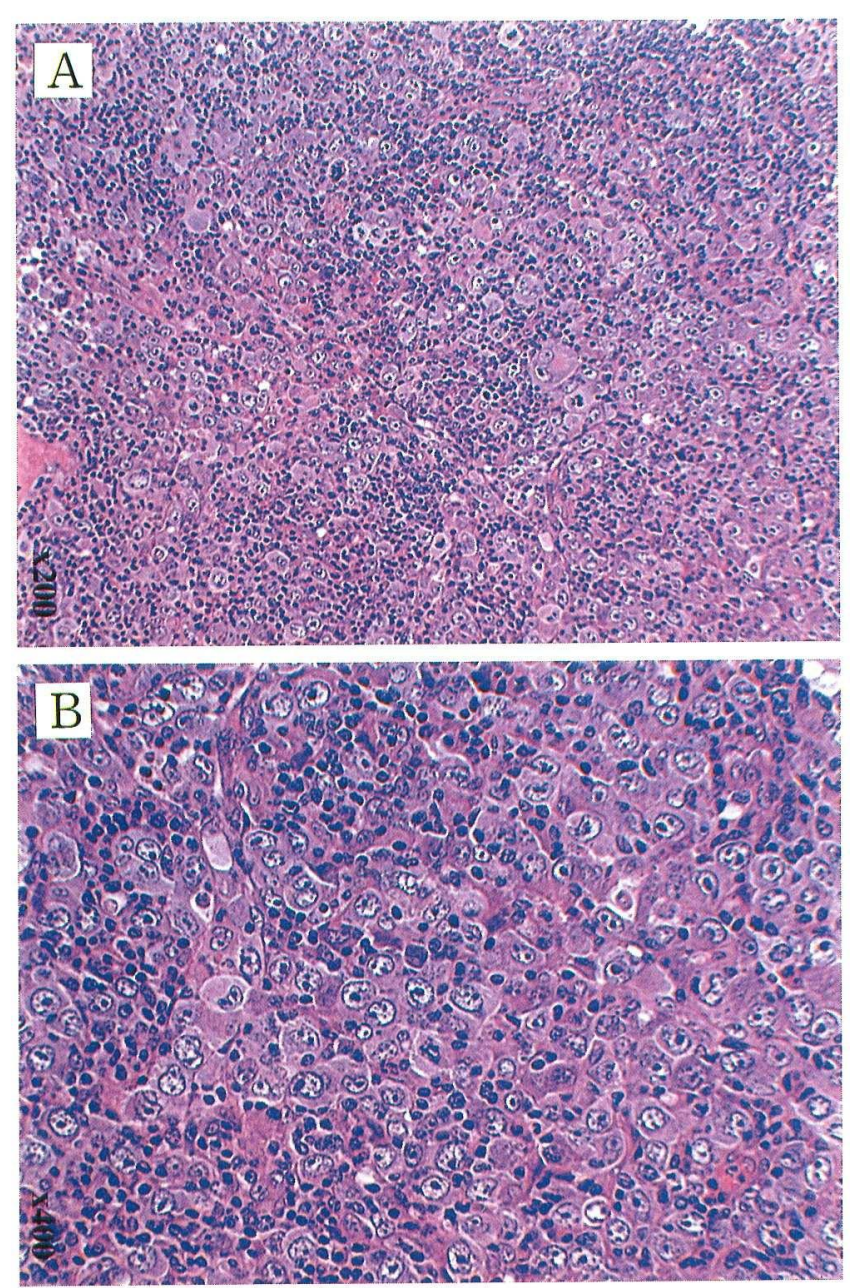

Figure 2. Large abnormal cells infiltrate the paracortical areas. They show convoluted round nuclei, large nucleoli and basophilic cytoplasm (HE stain, A $\times 200, B \times 400$ ).

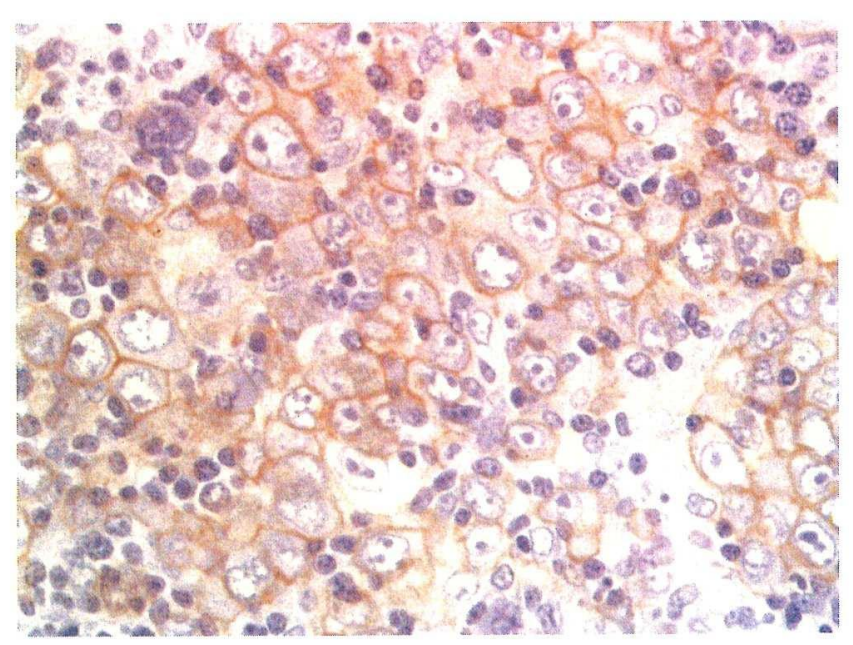

Figure 3. These abnormal cells are positive for Ki-1 (immunohistochemical stain, $\times 400$ ).

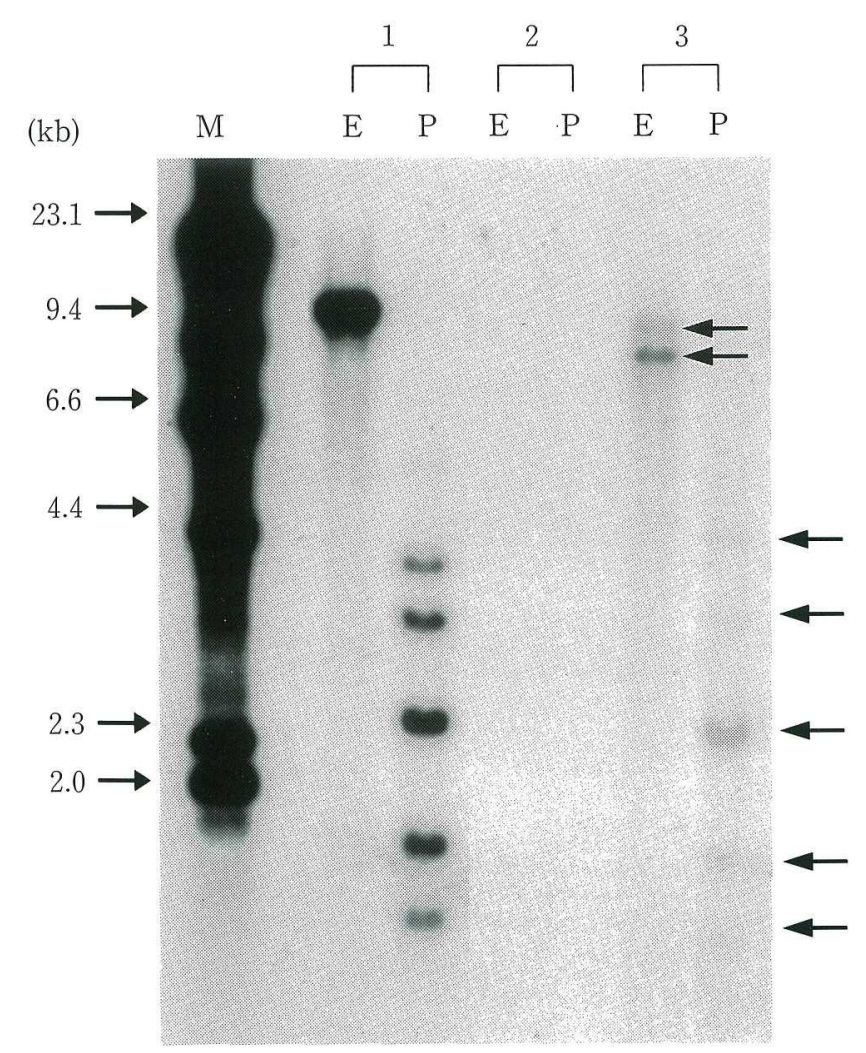

Figure 4. A Southern blot analysis of HTLV-1 proviral DNA. M: size marker, 1: positive control (monoclonal integrated DNA. Sequence size: $9043 \mathrm{~b}$ ), 2: negative control (human placental DNA), 3: patient's DNA, E: EcoRI digestion, P: PstI digestion, This patient's DNA shows two bands in EcoRI digestion and five bands in PstI digestion (arrows).

lymphoma (ALCL) based on the presence of positive CD30 (Ki-1) and intrasinusoidal growth patterns (2). ALCL is now regarded as a distinct clinicopathologic entity and has been included in the Revised European-American Lymphoma (REAL) Classification (3).

The CD30 ligand $(\mathrm{CD} 30 \mathrm{~L})$ is a transmembrane protein that belongs to the tumor necrosis factor (TNF) family (4). It can induce cell death in several CD30+ cell lines (5). Patients with CD30+ tumors frequently have elevated soluble CD30 (sCD30) levels in their serum, which correlate with a poor prognosis. Younes et al reported that blocking the apoptosis-inducing activity of CD30L by its soluble receptor may explain how CD30+ tumors escape immunosurveillance and may also be related to the reported poor prognosis of patients who have elevated sCD30 levels (6). However, the relationship between CD30+ ALCL and HTLV-1 infection has not yet been elucidated. In previous reports, this type of lymphoma, as observed in our patient, was reported in 2 to $5 \%$ of ATL patients (7-9). Clinically, this type of lymphoma has frequently shown extranodal tumors or nodules, but leukemic change, bone marrow involvement, and hypercalcemia are quite rare (8). Concerning ATL, extranodal tumor formation has rarely been reported $(10,11)$. 

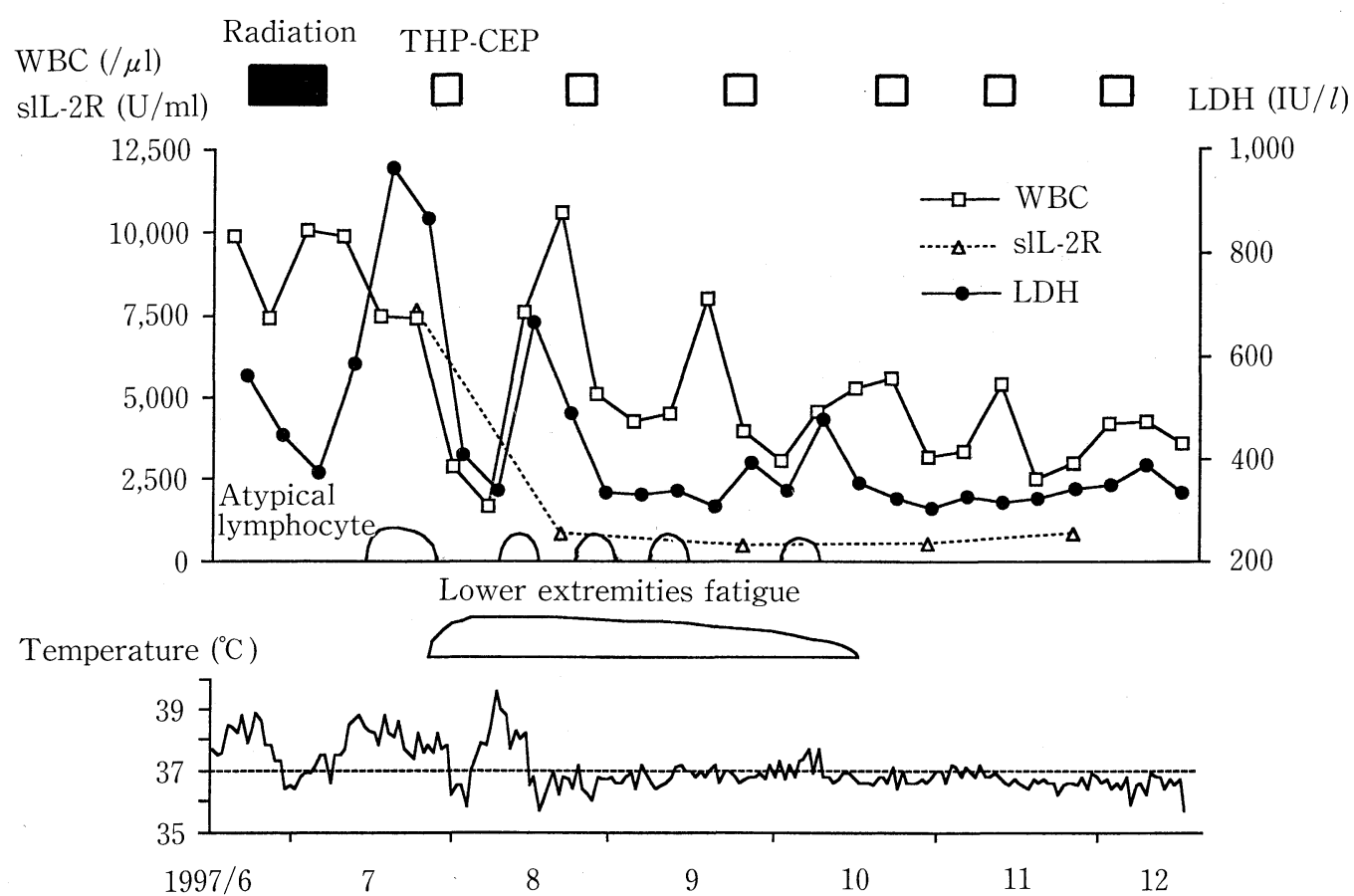

Figure 5. Clinical course. THP-CEP: pirarubicin; cyclophosphamide; etoposide; prednisolone, WBC: white blood cell, s1L-2R: soluble interleukin-2 receptor, LDH: lactate dehydrogenase.

The present patient demonstrated almost the same clinical findings and was also closely correlated with the previously reported cases of adult T-cell leukemia/lymphoma in which the pathohistological diagnosis was identical to that of $\mathrm{Ki}-1$ positive anaplastic large cell lymphoma.

Regarding the prognosis, Takeshita et al reported the median overall length of survival of CD30+ ALCL cases with HTLV-1 proviral DNA to be 11.9 months, while the 2- and 5year survival rates were $26 \%$ and $17 \%$, respectively (8). These rates are comensurate with the ATL lymphoma type (12). Tilly et al reported the event-free survival and overall survival to be better for ALCL than for non-ALCL (13). Pathogenetically the $\mathrm{p} 80$ expression activated by $\mathrm{t}(2 ; 5)(\mathrm{p} 23 ; \mathrm{q} 35)$ translocation has recently been shown to play an important role in the pathogenesis of ALCL (14). Nakamura et al reported p80 ALCL cases to constitute a very homogeneous group of tumors, characterized by their occurrence in a much younger age group (mainly in children and young adults with a median age of 17 years) and a relatively more favorable clinical course than the p80ALCL (15). Regarding the treatment for the present case, combination chemotherapy proved to be quite effective. Since the number of patients with ATL in which the pathohistological diagnosis was identical to that of CD30+ ALCL is small, the prognosis remains controversial. Therefore, the optimal regimen and the effectiveness of treatment for this type of lymphoma still needs to be elucidated in a larger number of patients with this disease.

\section{References}

1) Takatsuki $K$, Uchiyama $T$, Sagawa $K$, et al. Adult $T$ cell leukemia in Japan. in: Topics in Hematology, edited by S. Seno, F. Takaku and S. Irino, Amsterdam, Excerpta Medica, 1977: 73-77.

2) Stein H, Mason DY, Gerdes J, et al. The expression of the Hodgkin's disease associated antigen $\mathrm{Ki}-1$ in reactive and neoplastic lymphoid tissue: evidence that Reed-Sternberg cells and histiocytic malignancies are derived from activated lymphoid cells. Blood 66: 848-858, 1985.

3) Harris NL, Jaffe ES, Stein H, et al. A Revised European-American Classification of Lymphoid Neoplasm: A proposal From the International Lymphoma Study Group. Blood 84: 1361-1392, 1994.

4) Smith CA, Gruss HJ, Davis T, et al. CD30 antigen, a marker for Hodgkin's lymphoma, is a receptor whose ligand defines an emerging family of cytokines with homology to TNF. Cell 73: 1349-1360, 1993.

5) Gruss HJ, Boiani N, Williams DE, et al. Pleiotropic effects of the CD30 ligand on CD30-expressing cells and lymphoma cell lines. Blood 83: 2045-2056, 1994.

6) Younes A, Consoli U, Snell V, et al. CD30 ligand in Lymphoma Patients with CD30+ tumors. J Clin Oncol 15: 3355-3362, 1997.

7) Ohtsuka E, Kikuchi H, Nasu M, et al. Clinicopathological features of adult T-cell leukemia with CD 30 antigen expression. Leuk Lymphoma 15: 303-310, 1994.

8) Takeshita M, Ohshima K, Akamatsu M, et al. CD30-positive anaplastic large cell lymphoma in a human T-cell lymphotropic virus-I endemic area. Hum Pathol 26: 614-619, 1995.

9) Ohshima K, Kikuchi M, Masuda Y, et al. Defective provirus form of human T-cell leukemia virus type 1 in adult T-cell leukemia/lymphoma: Clinicopathological features. Cancer Res 51: 4639-4642, 1991.

10) Kikuchi M, Mitsui T, Takeshita M, et al. Virus associated adult T-cell leukemia (ATL) in Japan: clinical, histological and immunological studies. Hematol Oncol 4: 67-81, 1986.

11) Shimamoto Y, Yamaguchi M. HTLV-I induced extranodal lymphomas. 


\section{TAKAHARA et al}

Leuk Lymphoma 7: 37-45, 1992.

12) Taguchi H. An intensive chemotherapy of adult T-cell leukemia/lymphoma. Rinsho Ketsueki 37: 654-660, 1996.

13) Tilly H, Gaulard P, Lepage E, et al. Primary anaplastic large-cell lymphoma in adult: clinical presentation, immunophenotype, and outcome. Blood 90: 3727-3734, 1997.

14) Benz-Lemoine E, Brizard A, Huret JL, et al. Malignant histiocytosis: a specific $\mathrm{t}(2 ; 5)(\mathrm{p} 23 ; \mathrm{q} 35)$ translocation? Reviews of the literature. Blood 72: 1045-1047, 1988.

15) Nakamura $S$, Shiota $M$, Nakagawa A, et al. Anaplastic Large Cell Lymphoma: a distinct molecular pathologic entity: a reappraisal with special reference to p80 (NPM/ALK) expression. Am J Surg Pathol 21: 1420$1432,1997$. 